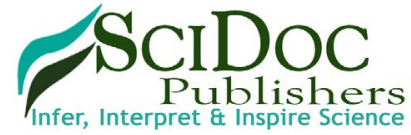

\section{Effect of Different Doses of Pregabalin on Erythrocyte Deformability in Rats with Lower Limb Ischemia Reperfusion Injury}

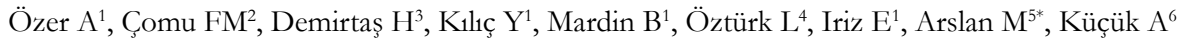

${ }^{1}$ Department of Cardiovascular Surgery, Gazi University Medical Faculty, Ankara, Turkey.

${ }^{2}$ Department of Physiology, Kırıkkale University Medical Faculty, Kirikkale, Turkey.

${ }^{3}$ Department of Cardiovascular Surgery, Yüksek Ihtisas Hospital, Kirikkale, Turkey.

${ }^{4}$ Department of Anaesthesiology and Reanimation, Yıldırım Beyazit University Medical Faculty, Ankara, Turkey.

${ }^{5}$ Department of Anaesthesiology and Reanimation, Gazi University Medical Faculty, Ankara, Turkey.

${ }^{6}$ Department of Physiology, Dumlupinar University Medical Faculty, Kütahya, Turkey.

\title{
Abstract
}

\begin{abstract}
Aim/Introduction: Acute ischemia reperfusion (IR) injury observed in the lower extremities occurs especially when a temporary cross-clamp is applied to the abdominal aorta during aortic surgery. Preoperative pregabalin has been used as a part of multimodal analgesia in postoperative pain treatment in recent years. Pregabalin has become one of the increasingly common agents in postoperative analgesia. In this study, we aimed to investigate the effect of pregabalin on erythrocyte deformability in rats undergoing IR.

Materials and Methods: 24 male Wistar albino rats weighing between 200-250 g were used in the study. Rats were randomly divided into 4 groups of 6 rats each (Control, Ischemia-Reperfusion (IR), IR-Pregabalin $50 \mathrm{mg}\left(50 \mathrm{mg} \cdot \mathrm{kg}^{-1}\right)$, IRPregabalin $200 \mathrm{mg}\left(200 \mathrm{mg} \cdot \mathrm{kg}^{-1}\right)$. Pregabalin was administered intraperitoneally $30 \mathrm{~min}$ before the procedure. An atraumatic microvascular clamp was placed across the infrarenal abdominal aorta in the IR groups. Following 120 min of ischemia, the clamp was removed and reperfusion was continued for $120 \mathrm{~min}$. All rats were euthanized by intraperitoneal administration of ketamine $\left(100 \mathrm{mg} \cdot \mathrm{kg}^{-1}\right)$ and taking blood from the abdominal aorta. Erythrocytes were seperated from heparinized whole blood samples. Deformability measurements were made in erythrocyte suspensions in phosphate buffered saline. A constant flow filtrometer system was used to measure erythrocyte deformability and relative resistance was calculated. Results: It was found that the formation of ischemia reperfusion increases the relative resistance according to the control group ( $\mathrm{p}<0.0001$ ). It was determined that application of pregabalin 50 or $200 \mathrm{mg}$ did not change erythrocyte deformability in ischemia reperfusion-induced rats $(\mathrm{p}=0.632, \mathrm{p}=0.811)$.

Conclusion: The administration of 50 or $200 \mathrm{mg}$ of pregabalin has no negative effect on the erythrocyte deformability in ischemia reperfusion-induced rats. We think that pregabalin can be safely used for analgesia in the cases of IR. However, these findings should be supported by clinical and experimental studies carried out in more detailed and broader series.
\end{abstract}

Keywords: Ischemia Reperfusion; Pregabalin; Different Dose; Erythrocyte Deformability.

\section{Introduction}

Cellular damage after reperfusion of previously viable ischaemic tissues in lower extremity is a common and critical clinical incident. Reperfusion of an acutely ischemic tissue may, paradoxically, lead to systemic complications that are associated with sig- nificant morbidity and mortality rates. As reperfusion progresses generally systemic inflammatory response syndrome and multiple organ failure (kidney, respiratory and circulatory system, etc.) follow local edema and muscle necrosis [1-5].

Pregabalin, a GABA analogue, is a potent new generation antiepi-

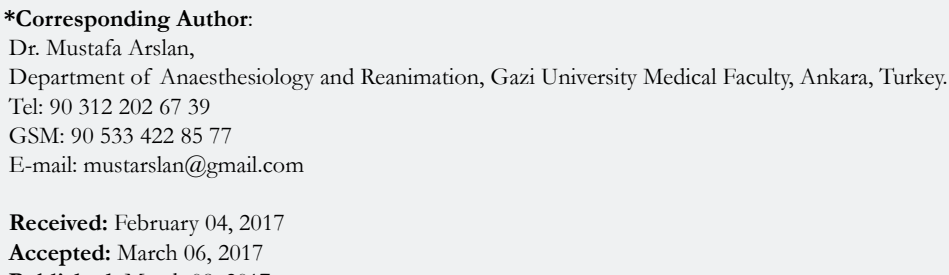

Citation: Özer A, Çomu FM, Demirtaş H, K1lıç Y, Arslan M, et al., (2017) Effect of Different Doses of Pregabalin on Erythrocyte Deformability in Rats with Lower Limb Ischemia Reperfusion Injury. Int J Anesth Res. 5(3), 414-416. doi: http:/ / dx.doi.org/10.19070/2332-2780-1700085

Copyright: Arslan $\mathbf{M}^{\circ}$ 2017. This is an open-access article distributed under the terms of the Creative Commons Attribution License, which permits unrestricted use, distri bution and reproduction in any medium, provided the original author and source are credited. 
leptic drug that is used for the treatment of partial onset seizures, painful diabetic neuropathy, and post-herpetic neuralgia [6]. It binds to the $\alpha 2 \delta$ subunit of the voltage-dependent calcium channel and does not bind to the protein. Pregabalin does not interact with liver enzymes and $95 \%$ of it is excreted by kidneys. Besides these clinical indications, it has some new properties that we need to focus on. Pregabalin has been used to protect against IR injury in many organs $[1,7,8]$.

Benefits of pregabalin use to prevent local and distal tissue injury due to IR has been well documented so far. However, not much is known about its protective effect on erythrocyte deformability following IR injury. The primary aim of this study was to investigate deformability changes and the role of pregabalin in preventing these changes in the erythrocytes of rats in an experimental model of lower limb muscle IR injury.

\section{Materials and Methods}

\section{Animals and Experimental Protocol}

This study was carried out in Gazi University Physiology Laboratory with the approval of the Ethics Committee of Experimental Animals of our university. All of the procedures were performed according to accepted standards of Guide for the Care and Use of Laboratory Animals.

In our study, 24 Wistar Albino rats weighing between 250 and 300 $\mathrm{g}$, raised under the same environmental conditions, were used. The rats were kept under $20-21^{\circ} \mathrm{C}$ at cycles of 12 -hour daylight and 12-hour darkness and had free access to food until 2 hours before the anesthesia procedure. The animals were randomly separated into four groups, each containing 6 rats. Midline laparatomy was done under general anesthesia.

Control group: A midline laparotomy was performed without any extra surgical intervention. After 2 hours of follow-up blood sample was collected and then animals were sacrificed.

I/R group: Midline laparotomy was performed in the same way. Infrarenal aorta was clamped for 2 hours. The clamp was removed and then reperfusion was started. Reperfusion lasted for two more hours. In the end after blood sampling from their abdominal aorta rats were sacrificed.

I/R group with pregabalin $\mathbf{5 0} \mathrm{mg}$ : Similiar steps were followed as mentioned above but in addition to the procedure pregabalin was given $\left(50 \mathrm{mg} \cdot \mathrm{kg}^{-1}\right)$ intraperitoneally for 30 minutes before ischemia period. After collecting blood samples rats were sacrificed at the end of 2 hours reperfusion period.

I/R group with pregabalin $200 \mathrm{mg}$ : Similiar steps were followed as mentioned above but in addition to the procedure pregabalin was given $\left(200 \mathrm{mg} \cdot \mathrm{kg}^{-1}\right)$ intraperitoneally for 30 minutes before ischemia period. After collecting blood samples rats were sacrificed at the end of 2 hours reperfusion period.

Rats were anesthetized with ketamine (100 mg. $\mathrm{kg}^{-1}$, intraperitoneally) and intracardiac blood samples were collected. Erythrocyte packs were prepared using heparinized total blood samples.
Erythrocytes packs were mixed with phosphate buffered saline (PBS) buffer to generate a suspension with the value of 5\% HCT. Those erythrocyte suspensions were used for the measurement of deformability.

\section{Deformability Measurements}

To prevent hemolysis samples of blood were collected very carefully and measurement process was done rapidly. Collected blood was centrifuged at $1000 \mathrm{rpm}$ for ten minutes. Blood plasma in the upper phase and the buffy coat, which is a thin layer of leukocytes mixed with platelets in the middle on erythrocytes were removed. Isotonic PBS buffer was added to collapsing erythrocytes and this mixture was centrifuged at $1000 \mathrm{rpm}$ for ten minutes. Liquid on the upper surface was taken. Washing process was repeated three times and finally pure red cell packs were obtained. Erythrocyte suspensions with $5 \%$ hematocrit in phosphate buffered saline (PBS) buffer were used to do deformability measurements. Erytrocytes were collected and then deformability measurements were done at $22^{\circ} \mathrm{C}$.

Erytrocytes deformability was measured by the constant-current filtrometre system. Samples were prepared as $10 \mathrm{ml}$ of erytrocytes suspension and PBS buffer before measurement. The infusion pump was set at $1.5 \mathrm{ml} / \mathrm{min}$ for a constant rate of flow. A $28 \mathrm{~mm}$ nucleoporin polycabonate filter with a $5 \mu \mathrm{m}$ pore diameter was used Consisting pressure changes while the erythrocytes passing through from the filter were detected by the pressure transducer and the data was transferred to computer with the help of MP 30 data equation systems (Biopac Systems Inc, Commat, USA). At different times pressure changes were measured by using relevant computer programs for calculations. Pressure calibration of the system was performed each time before measuring the samples. After buffer $\left(\mathrm{P}_{\mathrm{T}}\right)$ was passed through the filtration system the erythrocytes $\left(\mathrm{P}_{\mathrm{E}}\right)$ were passed secondly. Pressure variations were measured. By relating the pressure value of erythrocytes suspension to pressure value of buffer the relative refractory period value (Rrel) was calculated. The deformability index was interpreted as Rrel was increasing the ability of erythrocyte deformability was affected adversely.

\section{Statistical Analysis}

All the data were processed by variance analysis in SPSS 17.0 for Windows statistical software. A P-value less than 0.05 was considered statistically important. The data were expressed as mean \pm standard deviation. Variance analysis and Kruskal-Wallis test were used to evaluate the data. Mann Whitney $U$ test with Bonferroni correction were used to evaluate the variables with significance.

\section{Results}

The study showed that compared to control and IR groups relative resistance, a marker of erythrocyte deformability, was increased significantly by IR ( $p<0.0001)$, (Figure). The values of the control group were significantly lower than those of the IR, IR-P 50 and IR-P $200 \mathrm{mg}$ groups $(\mathrm{p}<0.0001, \mathrm{p}<0.0001, \mathrm{p}<$ 0.0001 , respectively). It was determined that application of pregabalin 50 or $200 \mathrm{mg}$ did not change erythrocyte deformability in IR-induced rats $(\mathrm{p}=0.632, \mathrm{p}=0.811$, respectively). 
Figure 1. Erythrocyte Deformability Index Values of the Groups. Each Bar Represents the mean \pm sd.

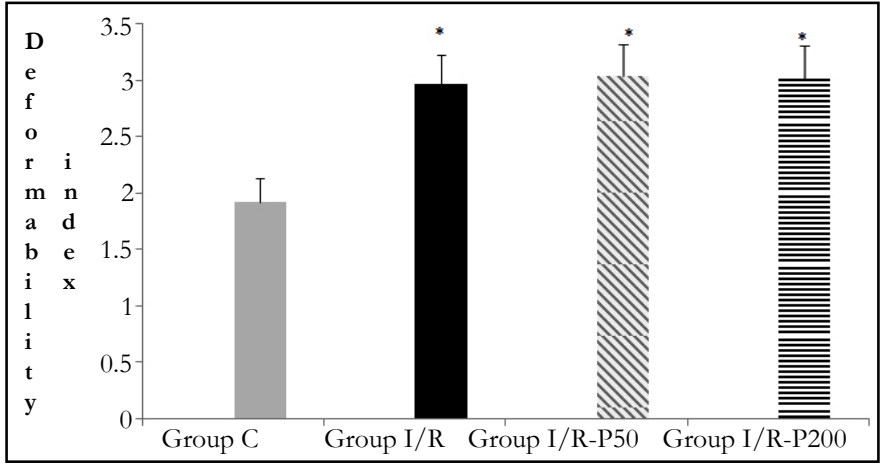

$* \mathrm{p}<0.05$ compared to the Group C

\section{Discussion}

Pregabalin is a structural analog of gamma-aminobutyric acid and has analgesic, anticonvulsant, anxiolytic and opioid-sparing effects. Pregabalin is a gabapentin derivative and has superior pharmacokinetic activity although it shows similar effect [8]. In animal studies, pregabalin, such as gabapentin, has also been shown to be effective in several models of neuropathic pain, incisional and inflammatory injury [9]. There are studies showing that preoperative administration of pregabalin reduces acute postoperative pain and analgesic consumption and the incidence of chronic neuropathic pain $[10,11]$.

Pregabalin is a gamma-amino-butyric acid analog with anticonvulsant, anxiolytic and opioid-sparing properties. In a study of epilepsy patients, oxcarbazepine has been shown to increase erythrocyte deformability at a shear force of $0.95 \mathrm{~Pa}$ [12].

For being capable for carrying oxygen and vital molecules to the final organ capillaries and excretion of metabolic wastes, erythrocytes must be able to extend and curve to move in these areas. This capacity, called as "deformability" is more important in microcirculation. Altered erythrocyte deformability changes both oxygen delivery capacity of the erythrocytes and the survival of the circulating erythrocytes [13-15].

In addition to these data our findings indicate that in rats undergoing IR erythrocyte deformability impairs and this impairment leads to disturbed microvascular perfusion and related problems. So we think that erythrocyte deformability measurement can be a useful parameter in IR injury. We also did not observe negative effect of pregabalin on maintaining erythrocyte deformability during periods of IR but we still think these promising results should further be supported by more detailed studies with larger volumes.

\section{References}

[1]. Millecamps M, Coderre TJ (2008) Rats with chronic post-ischemia pain exhibit an analgesic sensitivity profile similar to human patients with complex regional pain syndrome--type I. Eur J Pharmacol. 583(1): 97-102.

[2]. Duru S, Koca U, Oztekin S, Kar A, Coker C, et al., (2005) Antithrombin III pretreatment reduces neutrophil recruitment into the lung and skeletal muscle tissues in the rat model of biletaral lower limb and reperfusion: A pilot study. Acta Anaesthesiol Scand. 49(8): 1142-8.

[3]. Turchanyi B, Toth B, Racz I, Furesz J, Hamar J, et al., (2005) Ischemia reperfusion injury of skeletal muscle after selective deafferantation. Physiol Res. 54(1): 25-32.

[4]. Erer D, Özer A, Demirtaş H, Gönül II, Kara H, et al., (2016) Effects of Alprostadil and Iloprost on Renal, Lung and Skeletal Muscle Injury Following Hind limb Ischemia-Reperfusion Injury in Rats. Drug Des Devel Ther. 10: 2651-2658.

[5]. Erer D, Dursun AD, Oktar GL, Iriz E, Zor MH, et al., (2014) The effects of iloprost on lung injury induced by skeletal muscle ischemia-reperfusion. Bratisl Med J. 115 (7): 405-410.

[6]. Blommel ML, Blommel AL (2007) Pregabalin: An antiepileptic agent useful for neuropathic pain. Am J Health Syst Pharm. 64(14): 1475-82.

[7]. Kazanci B, Ozdogan S, Kahveci R, Gokce EC, Yigitkanli K, et al., (2016) Neuroprotective Effects of Pregabalin Against Spinal Cord IschemiaReperfusion Injury in Rats. Turk Neurosurg . doi: 10.5137/1019-5149. JTN.17959-16.1.

[8]. Ben-Menachem E (2004) Pregabalin pharmacology and its relevance to clinical practice. Epilepsia. 45(6): 13-8.

[9]. Gajraj NM (2007) Pregabalin: its pharmacology and use in pain management. Anesth Analg. 105(6): 1805-15.

[10]. Zhang J, Ho KY, Wang Y (2011) Efficacy of pregabalin in acute postoperative pain: a meta-analysis. Br J Anaesth. 106(4): 454-62.

[11]. Buvanendran A, Kroin JS, Della Valle CJ, Kari M, Moric M, et al., (2010) Perioperative oral pregabalin reduces chronic pain after total knee arthroplasty: a prospective, randomized, controlled trial. Anesth Analg. 110(1): 199-207.

[12]. Genç O, Erken G, Erken HA, Bolukbasi N, Bolukbasi O, et al., (2010) Epilepsili Hastalarda Antiepileptik Monoterapisinin Hemoreolojik parametreler Üzerindeki Etkisi. Türk Fizyolojik Bilimler Derneği 36. Ulusal Fizyoloji Kongresi. 18: 14-17.

[13]. Zinchuk VV (2001) Erythrocyte deformability: physiological aspects. Usp Fiziol Nauk. 32(3): 66-78.

[14]. Kuypers FA (1998) Red cell membrane damage. J Heart Valve Dis. 7: $387-$ 95.

[15]. Sivilotti ML (2004) Oxidant stres and haemolysis of the human erythrocyte. Toxicol Rev 23(3): 169-88. 